\title{
Solutions of Conformable Fractional-Order SIR Epidemic Model
}

\author{
Atimad Harir (D), Said Malliani, and Lalla Saadia Chandli \\ Laboratory of Applied Mathematics and Scientific Computing, Sultan Moulay Slimane University, P.O. Box 523,
} Beni Mellal 23000, Morocco

Correspondence should be addressed to Atimad Harir; atimad.harir@gmail.com

Received 18 October 2020; Revised 24 December 2020; Accepted 30 December 2020; Published 13 January 2021

Academic Editor: Giovanni P. Galdi

Copyright ( 2021 Atimad Harir et al. This is an open access article distributed under the Creative Commons Attribution License, which permits unrestricted use, distribution, and reproduction in any medium, provided the original work is properly cited.

In this paper, the conformable fractional-order SIR epidemic model are solved by means of an analytic technique for nonlinear problems, namely, the conformable fractional differential transformation method (CFDTM) and variational iteration method (VIM). These models are nonlinear system of conformable fractional differential equation (CFDE) that has no analytic solution. The VIM is based on conformable fractional derivative and proved. The result revealed that both methods are in agreement and are accurate and efficient for solving systems of OFDE.

\section{Introduction}

The problem of spreading of a (non) fatal disease in a population which is assumed to have a constant size over the period of the epidemic is considered in COVID-19. The goal of the epidemic model is to understand and if possible control the spread of disease [1]. At time $t$, suppose the population consists of

(i) $s(t)$ : the number of susceptible, who do not have the disease but could get it.

(ii) $i(t)$ : the number of infectives, who have the disease and can transmit it to others.

(iii) $r(t)$ : the number of removed, who cannot get the disease or transmit it; either they have a natural immunity or they have recovered from the disease and are immune from getting it again or they have been placed in isolation or they have died. The mathematical model does not distinguish between these possibilities.

Assume there is a steady constant rate between susceptible and infectives and that a constant proportion of this constant results in transmission. Then, in time $\delta \operatorname{tand} \delta s$, the susceptible becomes infective:

$$
\delta s=-\beta s i \delta t
$$

where $\beta$ is a positive constant. If $\gamma>0$ is the rate at which current infectives become isolated, then

$$
\delta i=\beta s i \delta t-\gamma i \delta t,
$$

and the number of new isolated $\delta r$ is given by

$$
\delta r=\gamma i \delta t
$$

If we let $\delta t \longrightarrow 0$, then the following nonlinear system of ODEs determines the progress of the disease:

$$
\left\{\begin{array}{l}
\frac{\mathrm{d}}{\mathrm{d} t} s(t)=-\beta s(t) i(t), \\
\frac{\mathrm{d}}{\mathrm{d} t} i(t)=\beta s(t) i(t)-\gamma i(t)=0, \\
\frac{\mathrm{d}}{\mathrm{d} t} r(t)=\gamma i(t),
\end{array}\right.
$$

with initial conditions

$$
\begin{aligned}
& s(0)=N_{s}, \\
& i(0)=N_{i}, \\
& r(0)=N_{r} .
\end{aligned}
$$

The following simple SIR model [2-5] is transformed to conformable fractional differential equation and is tested to 
show the efficiency of the variational iteration method [6] and differential transformation method [7-11] to solve such models.

Fractional differentiation and integration operators have different kinds of definitions which we can mention, the Riemann-Liouville definition $[12,13]$, the Caputo definition [14], and so on. Lately, Khalil et al. [15] introduced a new simple definition of the fractional derivative named the conformable fractional derivative (CFD), which can redress shortcomings of the other definitions. The main advantages of the CFD can be summarized as follows [16-19]:

(1) It satisfies all concepts and rules of an ordinary derivative such as: quotient, product, and chain rules, while the other fractional definitions fail to meet these rules

(2) It can be extended to solve exact and numerical fractional differential equations and systems easily and efficiently

The reason for considering a fractional-order system instead of its integer order counterpart is that fractionalorder differential equations are generalizations of integer order differential equations. Also, using fractional-order differential equations can help us to reduce the errors arising from the neglected parameters in modelling real-life phenomena.

We like to argue that fractional-order equations are more suitable than integer order ones in modelling biological, economical, and social systems (generally complex adaptive systems) where memory effects are important.

The main objective of our work is to introduce the conformable fractional-order approach for the study of a particular SIR model in a constant population. In this case, the conformable fractional-order system of the SIR model will be transformed to one conformable fractional equation and are solved using the variational iteration method and the conformable differential transformation method for numerical comparison.

\section{Conformable Fractional Derivative and Some Properties}

In this part, we review some definitions and some results of conformable fractional derivative. For more details, the reader can refer to $[15,18,20-25]$. The conformable fractional derivative of order $\alpha$ is defined as

$$
T_{\alpha} f(t)=\lim _{\varepsilon \longrightarrow 0} \frac{f\left(t+\varepsilon t^{1-\alpha}\right)-f(t)}{\varepsilon},
$$

for all $t>0, \alpha \in(0,1)$, and the fractional derivative at 0 is defined as

$$
\left(T_{\alpha} f\right)(0)=\lim _{t \longrightarrow 0^{+}}\left(T_{\alpha} f\right)(t) .
$$

Let $\alpha \in 0,1$ and $f$ be $\alpha$-differentiable at a point $t>0$, and if $f$ is differentiable, then

$$
T_{\alpha} f(t)=t^{1-\alpha} \frac{\mathrm{d}}{\mathrm{d} t} f(t)
$$

The fractional integral of order $\alpha$ is defined by

$$
I_{\alpha}(f)(t)=I\left(t^{\alpha-1} f\right)=\int_{0}^{t} s^{\alpha-1} f(s) \mathrm{d} s, \quad \text { for all } \alpha \in(0,1),
$$

where the integral is the usual Riemann improper integral.

\section{Mathematical Modeling of the Conformable Fractional SIR Model}

The conformable fractional model of actual evolution of this epidemic in a population of large size $N$ is given by the following conformable fractional differential system:

$$
\left\{\begin{array}{l}
T_{\alpha} s(t)=-\beta s(t) i(t), \\
T_{\alpha} i(t)=\beta s(t) i(t)-\gamma i(t), \\
T_{\alpha} r(t)=\gamma i(t),
\end{array}\right.
$$

with initial conditions

$$
\begin{aligned}
& s(0)=N_{s}, \\
& i(0)=N_{i}, \\
& r(0)=N_{r} .
\end{aligned}
$$

3.1. The Conformable Fractional Differential Transformation Method. In $[9,11]$, assume $f$ is infinitely $\alpha$-differentiable function, for some $0<\alpha \leq 1$. $f(t)$ can be expanded in fractional power series expansion about a point $t=0$ as

$$
f(t)=\sum_{k=0}^{\infty} \frac{t^{\alpha k}}{\alpha^{k} k !}\left[\left(T_{\alpha} f\right)^{(k)}\right]_{t=0}, \quad 0<t<R^{1 / \alpha}, R>0 .
$$

Here, $\left[\left(T_{\alpha} f\right)^{(k)}\right]_{t=0}$ denotes the application of the fractional derivative for $k$ times. Conformable fractional differential transform of $f(t)$ is defined as

$$
F_{\alpha}(k)=\frac{1}{\alpha^{k} k !}\left[\left(T_{\alpha} f\right)^{(k)}\right]_{t=0} .
$$

Then, the inverse conformable fractional differential transform of $F(k)$ is defined as

$$
f(t)=\sum_{k=0}^{\infty} t^{\alpha k} F_{\alpha}(k)=\sum_{k=0}^{\infty} \frac{t^{\alpha k}}{\alpha^{k} k !}\left[\left(T_{\alpha} f\right)^{(k)}\right]_{t=0} .
$$

The fundamental mathematical operations performed by conformable fractional differential transform method are listed.

\section{Proposition 1}

(i) If $f(t)=u(t) \pm v(t)$, then $F_{\alpha}(k)=U_{\alpha}(k) \pm V_{\alpha}(k)$.

(ii) If $f(t)=c u(t), c \in \mathbb{R}$, then $F_{\alpha}(k)=c U_{\alpha}(k)$.

(iii) If $f(t)=u(t) v(t)$, then $F_{\alpha}(k)=\sum_{l=0}^{k} U_{\alpha}(l) V_{\alpha}$ $(k-l)$ 
(iv) If $f(t)=T_{\alpha} u(t)$, then $F_{\alpha}(k)=\alpha(k+1) U_{\alpha}(k+1)$.

(v) If $f(t)=\left(t-t_{0}\right)^{p}$, then $F_{\alpha}(k)=\delta(k-(p / \alpha))$, where

$$
\delta(k)= \begin{cases}1, & \text { if } k=0 \\ 0, & \text { if } k \neq 0\end{cases}
$$

3.1.1. Application of Conformable Fractional Differential Transform Method. Equation (10) can be rewritten as follows:

$$
\left\{\begin{array}{l}
\alpha(k+1) S_{\alpha}(t)=-\beta \sum_{l=0}^{k} S_{\alpha}(l) I_{\alpha}(k-l), \\
\alpha(k+1) I_{\alpha}(t)=\beta \sum_{l=0}^{k} S_{\alpha}(l) I_{\alpha}(k-l)-\gamma I_{\alpha}(k), \\
\alpha(k+1) R_{\alpha}(t)=\gamma I_{\alpha}(k) .
\end{array}\right.
$$

Hence, recurrence relation is obtained as

$$
\left\{\begin{array}{l}
S_{\alpha}(t)=\frac{1}{\alpha(k+1)}\left[-\beta \sum_{l=0}^{k} S_{\alpha}(l) I_{\alpha}(k-l)\right], \\
I_{\alpha}(t)=\frac{1}{\alpha(k+1)}\left[\beta \sum_{l=0}^{k} S_{\alpha}(l) I_{\alpha}(k-l)-\gamma I_{\alpha}(k)\right], \\
R_{\alpha}(t)=\frac{1}{\alpha(k+1)}\left[\gamma I_{\alpha}(k)\right] .
\end{array}\right.
$$

With initial conditions $N_{s}=2000, N_{i}=300$, and $N_{r}=$ 200 and parameters $N=2500, \beta=0.00012$, and $\gamma=0.1$, apply the condition in (17); then, the closed form of the solution where $k=4$ can be written as

$$
\begin{aligned}
s(t)= & \sum_{l=0}^{k} S(k) t^{k \alpha}=2000-72 \frac{t^{\alpha}}{\alpha}-3.744 \frac{t^{2 \alpha}}{\alpha^{2}}+0.034368 \frac{t^{3 \alpha}}{\alpha^{3}} \\
& +0.013309 \frac{t^{4 \alpha}}{\alpha^{4}}, \\
i(t)= & \sum_{l=0}^{k} I(k) t^{k \alpha}=300+42 \frac{t^{\alpha}}{\alpha}+1.644 \frac{t^{2 \alpha}}{\alpha^{2}}-0.089168 \frac{t^{3 \alpha}}{\alpha^{3}} \\
& -0.011080 \frac{t^{4 \alpha}}{\alpha^{4}}, \\
r(t)= & \sum_{l=0}^{k} R(k) t^{k \alpha}=200+30 \frac{t^{\alpha}}{\alpha}+2.1 \frac{t^{2 \alpha}}{\alpha^{2}}+0.0548 \frac{t^{3 \alpha}}{\alpha^{3}} \\
& -0.002229 \frac{t^{4 \alpha}}{\alpha^{4}} .
\end{aligned}
$$

3.2. Variational Iteration Method. To illustrate the basic idea of the variational iteration method, we consider the following nonlinear differential equation in the operator form:

$$
L(u(t))+N(u(t))=g(t),
$$

where $L$ is a linear operator, $N$ is a nonlinear operator, and $g$ is any real function which is called the inhomogeneous term. Then, the corresponding correction functional for equation (19) is given by

$$
u_{n+1}(t)=u_{n}(t)+\int_{0}^{t} \lambda\left\{L u_{n}(s)+N \widetilde{u}_{n}(s)-g(s)\right\} \mathrm{d} s,
$$

where $\lambda$ is the general Lagrange multiplier [26], which can be identified optimally via the variational theory [27] and $N \tilde{u}_{n}$ is considered as restricted variation, i.e., $\delta N \widetilde{u}_{n}=0$. Consider the stationary condition of the above correction functional.

Theorem 1. Consider the conformable fractional differential equation (10). Then, the variational iteration formula is given by

$$
\left\{\begin{array}{l}
s_{n+1}(t)=s_{n}(t)-I_{\alpha}\left\{T_{\alpha} s_{n}(t)+\beta s_{n}(t) i_{n}(t)\right\}, \\
i_{n+1}(t)=i_{n}(t)-I_{\alpha}\left\{T_{\alpha} i_{n}(t)-\beta s_{n}(t) i_{n}(t)+\gamma i_{n}(t)\right\}, \\
r_{n+1}(t)=r_{n}(t)-I_{\alpha}\left\{T_{\alpha} r_{n}(t)-\gamma i_{n}(t)\right\},
\end{array}\right.
$$

where $s_{n}, i_{n}$, and $r_{n}$ are the $n$th approximation, $T_{\alpha}$ is the conformable fractional derivative of order $\alpha$, and $I_{\alpha}$ is the fractional integral of order $\alpha \in 0,1$.

Proof. Equation (10) may be rewritten equivalently as

$$
\left\{\begin{array}{l}
T_{\alpha} s_{n}(t)+\beta s_{n}(t) i_{n}(t)=0, \\
T_{\alpha} i_{n}(t)-\beta s_{n}(t) i_{n}(t)+\gamma i_{n}(t)=0, \\
T_{\alpha} r_{n}(t)-\gamma i_{n}(t)=0 .
\end{array}\right.
$$

Multiplying equation (22) by a general Lagrange multiplier $\lambda_{1}(t), \lambda_{2}(t)$, and $\lambda_{3}(t)$ yields

$$
\left\{\begin{array}{l}
\lambda_{1}(t)\left\{T_{\alpha} s_{n}(t)+\beta s_{n}(t) i_{n}(t)\right\}=0, \\
\lambda_{2}(t)\left\{T_{\alpha} i_{n}(t)-\beta s_{n}(t) i_{n} t+\gamma i_{n}(t)\right\}=0, \\
\lambda_{3}(t)\left\{T_{\alpha} r_{n}(t)-\gamma i_{n}(t)\right\}=0 .
\end{array}\right.
$$

Now, upon applying $I_{\alpha}$ to the both sides of equation (23) will give

$$
\left\{\begin{array}{l}
I_{\alpha}\left[\lambda_{1}(t)\left\{T_{\alpha} s_{n}(t)+\beta s_{n}(t) i_{n}(t)\right\}\right]=0, \\
I_{\alpha}\left[\lambda_{2}(t)\left\{T_{\alpha} i_{n}(t)-\beta s_{n}(t) i_{n}(t)+\gamma i_{n}(t)\right\}\right]=0, \\
I_{\alpha}\left[\lambda_{3}(t)\left\{T_{\alpha} r_{n}(t)-\gamma i_{n}(t)\right\}\right]=0 .
\end{array}\right.
$$

Then, the correction functional of equation (10) will be read as follows:

$$
\begin{aligned}
& s_{n+1}(t)=s_{n}(t)+I_{\alpha}\left[\lambda_{1}(t)\left\{T_{\alpha} s_{n}(t)+\beta \widetilde{s}_{n}(t) \tilde{i}_{n}(t)\right\}\right], \\
& i_{n+1}(t)=i_{n}(t)+I_{\alpha}\left[\lambda_{2}(t)\left\{T_{\alpha} i_{n}(t)-\beta \widetilde{s}_{n}(t) \widetilde{i}_{n}(t)+\gamma \tilde{i}_{n}(t)\right\}\right], \\
& r_{n+1}(t)=r_{n}(t)+I_{\alpha}\left[\lambda_{3}(t)\left\{T_{\alpha} r_{n}(t)-\gamma \tilde{i}_{n}(t)\right\}\right] .
\end{aligned}
$$


In this case, the value of $\lambda_{j}(t)$, for $j=\{1,2,3\}$, cannot be evaluated easily from equation (25), which will be a functional with fractional integral. Equation (25) can be expressed as follows:

$$
\begin{aligned}
& s_{n+1}(t)=s_{n}(t)+\int_{0}^{t} \tau^{\alpha-1}\left[\lambda_{1}(\tau)\left\{\tau^{1-\alpha} \frac{\mathrm{d}}{\mathrm{d} \tau} s_{n}(\tau)+\beta \widetilde{s}_{n}(\tau) \widetilde{i}_{n}(\tau)\right\}\right] \mathrm{d} \tau, \\
& i_{n+1}(t)=i_{n}(t)+\int_{0}^{t} \tau^{\alpha-1}\left[\lambda_{2}(\tau)\left\{\tau^{1-\alpha} \frac{\mathrm{d}}{\mathrm{d} \tau} i_{n}(\tau)-\beta \widetilde{s}_{n}(\tau) \widetilde{i}_{n}(\tau)+\gamma \widetilde{i}_{n}(\tau)\right\}\right] \mathrm{d} \tau, \\
& r_{n+1}(t)=r_{n}(t)+\int_{0}^{t} \tau^{\alpha-1}\left[\lambda_{3}(\tau)\left\{\tau^{1-\alpha} \frac{\mathrm{d}}{\mathrm{d} \tau} r_{n}(\tau)-\gamma \widetilde{i}_{n}(\tau)\right\}\right] \mathrm{d} \tau,
\end{aligned}
$$

where $\widetilde{s}_{n}$ and $\widetilde{i}_{n}$ are the restricted variations with $\delta \widetilde{s}_{n}=0$ and $\delta \widetilde{i}_{n}=0$. From equation (26), we obtain

$$
\begin{aligned}
& \delta s_{n+1}(t)=\delta s_{n}(t)+\delta \int_{0}^{t}\left[\lambda_{1}(\tau)\left\{\frac{\mathrm{d}}{\mathrm{d} \tau} s_{n}(\tau)+\beta \tau^{\alpha-1} \widetilde{s}_{n}(\tau) \widetilde{i}_{n}(\tau)\right\}\right] \mathrm{d} \tau \\
& \delta i_{n+1}(t)=\delta i_{n}(t)+\delta \int_{0}^{t}\left[\lambda_{2}(\tau)\left\{\frac{\mathrm{d}}{\mathrm{d} \tau} i_{n}(\tau)-\beta \tau^{\alpha-1} \widetilde{s}_{n}(\tau) \widetilde{i}_{n}(\tau)+\gamma \tau^{\alpha-1} \widetilde{i}_{n}(\tau)\right\}\right] \mathrm{d} \tau \\
& \delta r_{n+1}(t)=\delta r_{n}(t)+\delta \int_{0}^{t}\left[\lambda_{3}(\tau)\left\{\frac{\mathrm{d}}{\mathrm{d} \tau} r_{n}(\tau)-\gamma \tau^{\alpha-1} \widetilde{i}_{n}(\tau)\right\}\right] \mathrm{d} \tau
\end{aligned}
$$

Using integration by parts, equation (27) becomes (for equations (28) and (29) are similar to the proof of equation (27))

$$
\begin{aligned}
\delta s_{n+1}(t) & =\delta s_{n}(t)+\delta\left(\lambda(t) s_{n}(t)-\int_{0}^{t} \lambda_{1}^{\prime}(\tau) s_{n}(\tau) \mathrm{d} \tau\right) \\
& =\left(1+\lambda_{1}(t)\right) \delta s_{n}(t)-\delta \int_{0}^{t} \lambda_{1}^{\prime}(\tau) s_{n}(\tau) \mathrm{d} \tau, \\
\delta i_{n+1}(t) & =\left(1+\lambda_{2}(t)\right) \delta i_{n}(t)-\delta \int_{0}^{t} \lambda_{2}^{\prime}(\tau) i_{n}(\tau) \mathrm{d} \tau, \\
\delta r_{n+1}(t) & =\left(1+\lambda_{3}(t)\right) \delta r_{n}(t)-\delta \int_{0}^{t} \lambda_{3}^{\prime}(\tau) r_{n}(\tau) \mathrm{d} \tau .
\end{aligned}
$$

The Lagrange multipliers $\lambda_{1}(t), \lambda_{2}(t)$, and $\lambda_{3}(t)$ can be obtained by $\lambda_{j}^{\prime}(\tau)=0$ for all $j=\{1,2,3\}$ with boundary condition: $1+\lambda_{j}(t)=0$, for all $j=\{1,2,3\}$. Solving the last initial value problem for $\lambda_{j}$ for all $j=\{1,2,3\}$, the general Lagrange multiplier $\lambda_{j}$ is found to be

$$
\lambda_{j}=-1, \quad \text { for all } j=\{1,2,3\} \text {. }
$$

Hence, substituting the value of $\lambda_{j}$ into the corresponding correction functional (25) will give the following iteration formula:

$$
\begin{aligned}
& s_{n+1}(t)=s_{n}(t)-I_{\alpha}\left\{T_{\alpha} s_{n}(t)+\beta \widetilde{s}_{n}(t) \widetilde{i}_{n}(t)\right\}, \\
& i_{n+1}(t)=i_{n}(t)-I_{\alpha}\left\{T_{\alpha} i_{n}(t)-\beta \widetilde{s}_{n}(t) \widetilde{i}_{n}(t)+\gamma \widetilde{i}_{n}(t)\right\}, \\
& r_{n+1}(t)=r_{n}(t)-I_{\alpha}\left\{T_{\alpha} r_{n}(t)-\gamma \widetilde{i}_{n}(t)\right\} .
\end{aligned}
$$

3.2.1. Application of the Variational Iteration Method. Applying the variational iteration method using Theorem 1,

$$
\begin{aligned}
& s_{n+1}(t)=s_{n}(t)-I_{\alpha}\left\{T_{\alpha} s_{n}(t)+\beta \widetilde{s}_{n}(t) \widetilde{i}_{n}(t)\right\}, \\
& i_{n+1}(t)=i_{n}(t)-I_{\alpha}\left\{T_{\alpha} i_{n}(t)-\beta \widetilde{s}_{n}(t) \widetilde{i}_{n}(t)+\gamma \widetilde{i}_{n}(t)\right\}, \\
& r_{n+1}(t)=r_{n}(t)-I_{\alpha}\left\{T_{\alpha} r_{n}(t)-\gamma \widetilde{i}_{n}(t)\right\} .
\end{aligned}
$$

With initial conditions $N_{s}=2000, N_{i}=300$, and $N_{r}=$ 200 and parameter $N=2500, \beta=0.00012$, and $\gamma=0.1$, we obtain 


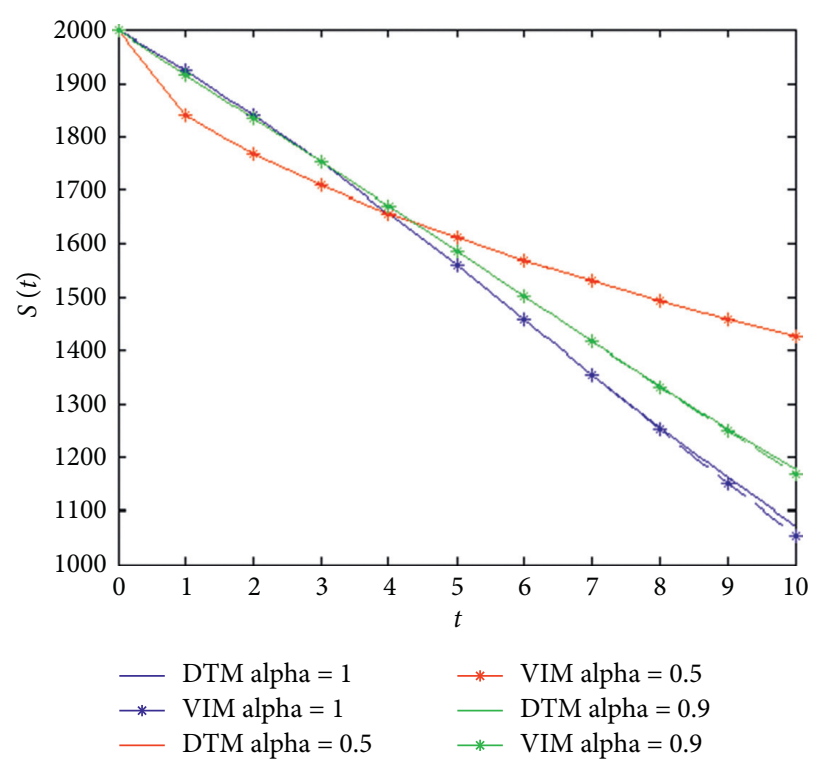

FIgure 1: Comparison of VIM solutions with the CDTM solutions of $S(t)$ for system (10) at different $\alpha$.

$$
\begin{aligned}
& s_{1}(t)=2000-72 \frac{t^{\alpha}}{\alpha}, \\
& i_{1}(t)=300+42 \frac{t^{\alpha}}{\alpha} \\
& r_{1}(t)=200+30 \frac{t^{\alpha}}{\alpha} \\
& s_{2}(t)=2000-72 \frac{t^{\alpha}}{\alpha}-3.744 \frac{t^{2 \alpha}}{\alpha^{2}}+0.12096 \frac{t^{3 \alpha}}{\alpha^{3}}, \\
& i_{2}(t)=300+42 \frac{t^{\alpha}}{\alpha}+1.644 \frac{t^{2 \alpha}}{\alpha^{2}}-0.12096 \frac{t^{3 \alpha}}{\alpha^{3}}, \\
& r_{2}(t)=200+30 \frac{t^{\alpha}}{\alpha}+2.1 \frac{t^{2 \alpha}}{\alpha^{2}} \\
& s_{3}(t)=2000-72 \frac{t^{\alpha}}{\alpha}-3.744 \frac{t^{2 \alpha}}{\alpha^{2}}+0.034368 \frac{t^{3 \alpha}}{\alpha^{3}}+0.014437 \frac{t^{4 \alpha}}{\alpha^{4}}-0.000183 \frac{t^{5 \alpha}}{\alpha^{5}} \\
& -0.000013 \frac{t^{6 \alpha}}{\alpha^{6}}+2.51 * 10^{-7} \frac{t^{7 \alpha}}{\alpha^{7}} \\
& i_{3}(t)=300+42 \frac{t^{\alpha}}{\alpha}+1.644 \frac{t^{2 \alpha}}{\alpha^{2}}-0.089168 \frac{t^{3 \alpha}}{\alpha^{3}}-0.014413 \frac{t^{4 \alpha}}{\alpha^{4}}+0.000183 \frac{t^{5 \alpha}}{\alpha^{5}} \\
& +0.000013 \frac{t^{6 \alpha}}{\alpha^{6}}+2.51 * 10^{-7} \frac{t^{7 \alpha}}{\alpha^{7}} \\
& r_{3}(t)=200+30 \frac{t^{\alpha}}{\alpha}+2.1 \frac{t^{2 \alpha}}{\alpha^{2}}+0.0548 \frac{t^{3 \alpha}}{\alpha^{3}}-0.003024 \frac{t^{4 \alpha}}{\alpha^{4}} .
\end{aligned}
$$




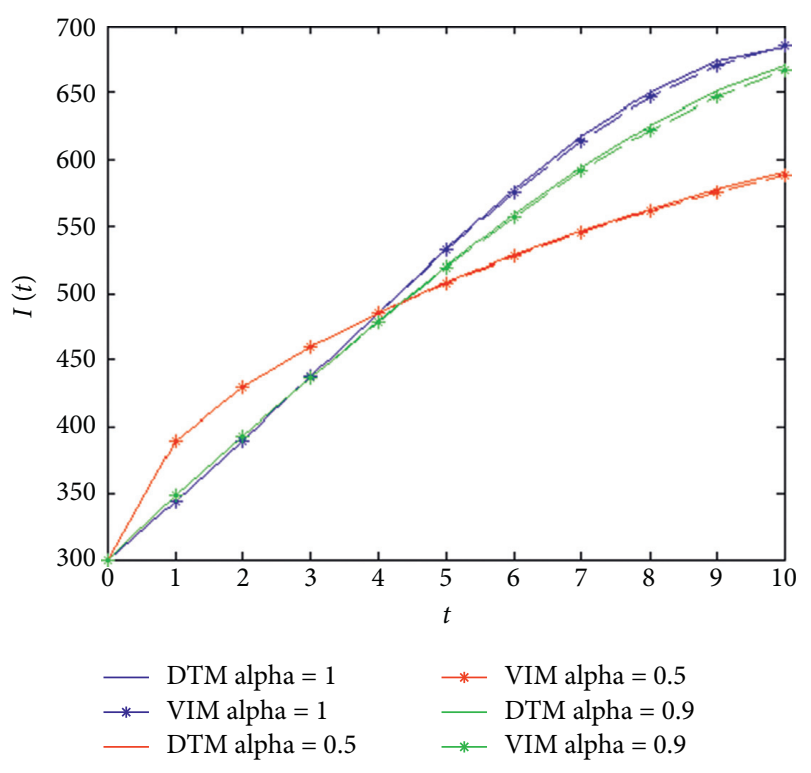

FIgUre 2: Comparison of VIM solutions with the CDTM solutions of $I(t)$ for system (10) at different $\alpha$.

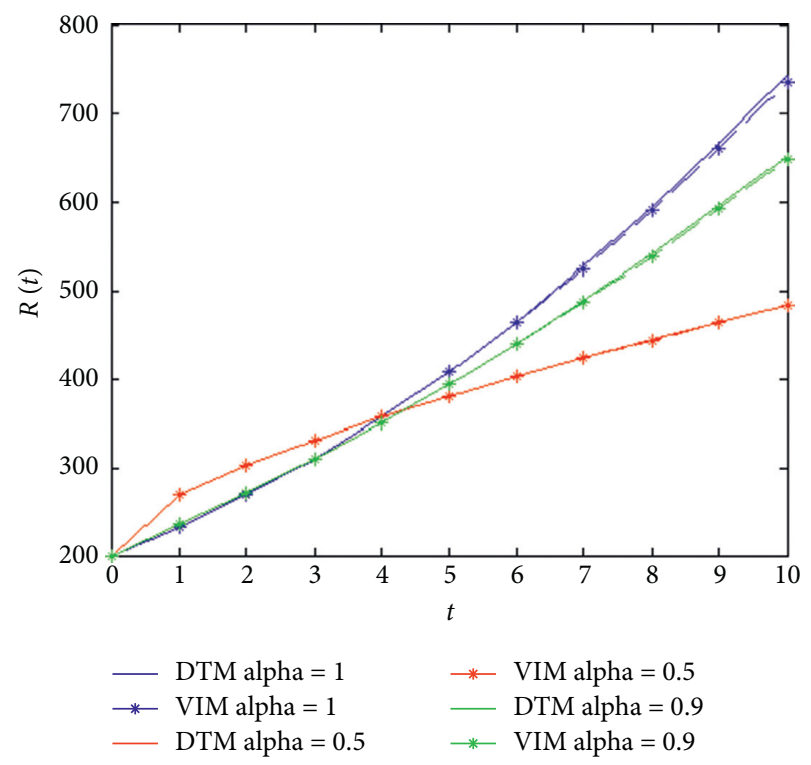

FIgURE 3: Comparison of VIM solutions with the CDTM solutions of $R(t)$ for system (10) at different $\alpha$.

It can be observed that the result of the epidemic system of equation (10) is in complete agreement with the result obtained by the conformable fractional differential transformation method. Figures 1, 2 and 3.

\section{Conclusion}

In this study, we have found out approximate solutions with two numerical methods for the SIR epidemic model. These methods are based on conformable derivative which is extremely popular in the last years. Firstly, by using the $\alpha$-derivative, we have redefined the conformable differential transformation method (CDTM) and variational iteration method (VIM). Then, we have demonstrated the efficiencies and accuracies of the proposed methods by applying them to the SIR epidemic model. It is found that the approximate solution generated the VIM by our method which is in complete agreement with the corresponding approximate solution CDTM. Besides, in view of their usability, our methods are applicable to many epidemic models SEIR/SEIRS and SIS of fractional order.

\section{Data Availability}

No data were used to support this study.

\section{Conflicts of Interest}

The authors declare that they have no conflicts of interest.

\section{References}

[1] W. Kermack and A. Mckendrick, "A contribution to the mathematical theorynof epidemics," Proceedings of the Royal Society A, vol. 115, pp. 700-721, 1927.

[2] please provide the complete reference details query has been raised..

[3] S. Gao, Z. Teng, and D. Xie, "Analysis of a delayed SIR epidemic model with pulse vaccination," Chaos, Solitons \& Fractals, vol. 40, no. 2, pp. 1004-1011, 2009.

[4] J. Biazer, "Solution of the epidemic model by Adomain decomposition method," Applied Mathematics and Computation, vol. 173, pp. 1101-1106, 2006.

[5] J. Satsuma, R. Willox, A. Ramani, B. Grammaticos, and A. S. Carstea, "Extending the SIR epidemic model," Physica A: Statistical Mechanics and Its Applications, vol. 336, no. 3-4, pp. 369-375, 2004.

[6] M. Rafei, H. Daniali, and D. D. Ganji, "Variational iteration method for solving the epidemic model and the prey and predator problem," Applied Mathematics and Computation, vol. 186, no. 2, pp. 1701-1709, 2007.

[7] I. H. Abdel-Halim Hassan, "Application to differential transformation method for solving systems of differential equations," Applied Mathematical Modelling, vol. 32, no. 12, pp. 2552-2559, 2008.

[8] M. Farshid, "Differential transform method for solving linear and nonlinear systems of ordinary differential equations," Applied Mathematical Sciences, vol. 70, pp. 3465-3472, 2011.

[9] E. Ünal and A. Gökdoğan, "Solution of conformable fractional ordinary differential equations via differential transform method," Optik, vol. 128, pp. 264-273, 2017.

[10] F. Evirgen, "Analyze the optimal solutions of optimization problems by means offractional gradient based system using VIM," An International Journal of Optimization and Control: Theories and Applications, vol. 6, no. 2, pp. 75-83, 2016.

[11] A. Harir, S. Melliani, H. El Harfi, and L. S. Chadli, "Variational iteration method and differential transformation method for solving the SEIR epidemic model," International Journal of Differential Equations, vol. 2020, Article ID 3521936, 7 pages, 2020.

[12] Ks. Miller and B. Ross, An Introduction to the Fractional Calculus and Fractional Differential Equations, Wiley, New York, NY, USA, 1993.

[13] A. Harir, S. Melliani, and L. S. Chadli, "Fuzzy fractional evolution equations and fuzzy solution operators," Advances 
in Fuzzy Systems, vol. 2019, Article ID 5734190, 10 pages, 2019.

[14] I. Podlubny, Fractional differential equations an introduction to fractional derivatives, fractional differential equations, to methods of their solution and some of their applications, Academic Press, Cambridge, MA, USA, 1998.

[15] R. Khalil, M. Al Horani, A. Yousef, and M. Sababheh, "A new definition of fractional derivative," Journal of Computational and Applied Mathematics, vol. 264, pp. 65-70, 2014.

[16] B. B. Iskender Eroglu, D. Avci, and N. Ozdemir, "Constrained optimal control of a fractionally damped elastic beam," International Journal of Nonlinear Sciences and Numerical Simulation, vol. 21, no. 3, 4 pages, 2020.

[17] M. Yavuz, "Novel solution methods for initial boundary value problems of fractional order with conformable differentiation," An International Journal of Optimization and Control: Theories and Applications (IJOCTA), vol. 8, no. 1, 7 pages, 2018.

[18] B. B. Iskender Eroglu and N. Ozdemir, "Conformable heat equation on a radial. symmetric plate," Thermal Science, vol. 21, no. 2, pp. 819-826, 2017.

[19] A. Hamdachea, S. Saadib, and I. Elmouki, "Free terminal time optimal control problem for the treatment of HIV infection," An International Journal of Optimization and Control: Theories and Applications, vol. 6, no. 1, pp. 33-51, 2016.

[20] T. Abdeljawad, "On conformable fractional calculus," Journal of Computational and Applied Mathematics, vol. 279, pp. 57-66, 2015.

[21] A. A. Al-shawba, K. A. Gepreel, F. A. Abdullah, and A. Azmi, "Abundant closed form solutions of the conformable time fractional sawada-kotera-Ito equation using ( $\left.G^{\prime} / G\right)$-expansion method," Results in Physics, vol. 9, pp. 337-343, 2018.

[22] M. Eslami and H. Rezazadeh, "The first integral method for Wu-Zhang system with conformable time-fractional derivative," Calcolo, vol. 53, no. 3, pp. 475-485, 2016.

[23] A. Harir, S. Melliani, and L. S. Chadli, "Fuzzy generalized conformable fractional derivative," Advances in Fuzzy Systems, Advances in Fuzzy Systems, vol. 2020, p. 7, Article ID 1954975, 2020.

[24] B. B. Iskender Eroglu, D. Avci, and N. Ozdemir, "Optimal control problem for a conformable fractional heat conduction equation," Acta Physica Polonica A, vol. 132, no. 3, pp. 658662, 2017.

[25] F. Evirgen, "Conformable fractional gradient based dynamic system for constrained optimization problem," Acta Physica Polonica A, vol. 132, no. 3-II, pp. 1066-1069, 2017.

[26] M. Inokuti, "General use of the lagrange multiplier in nonliniear mathematical physics," in Variational Method in the Mechanics of Solids, S. Nemat-Nassed, Ed., pp. 156-162, Pergemon Press, Oxford, UK, 1978.

[27] J.-H. He, "Some asymptotic methods for strongly nonlinear equations," International Journal of Modern Physics B, vol. 20, no. 10, pp. 1141-1199, 2006. 